\title{
Cerebral Infarction After Lobectomy for Lung Cancer
}

\author{
Hiroyuki Miura $^{1,}$, Jun Miura $^{2}$, Hiroshi Hirano ${ }^{3}$ \\ ${ }^{1}$ Department of Thoracic Surgery, Akiru Municipal Medical Center, Akiruno City, Tokyo, Japan \\ ${ }^{2}$ Department of Surgery, Kyorin University School of Medicine, Mitaka City, Tokyo, Japan \\ ${ }^{3}$ Department of Pathology, Hachioji Medical Center of Tokyo Medical University, Hachioji City, Tokyo, Japan
}

Email address:

arowana@akiru-med.jp (H. Miura)

${ }^{*}$ Corresponding author

\section{To cite this article:}

Hiroyuki Miura, Jun Miura, Hiroshi Hirano. Cerebral Infarction After Lobectomy for Lung Cancer. Journal of Surgery. Vol. 7, No. 3, 2019, pp. 63-66. doi: 10.11648/j.js.20190703.13

Received: April 22, 2019; Accepted: May 28, 2019; Published: June 10, 2019

\begin{abstract}
Background and objective: The left upper lobectomy as treatment for lung cancer has been identified as a risk factor for cerebral infarction. We analyzed cases of surgery for lung cancer to clarify factors that increase the risk for postoperative cerebral infarction. Methods: This study included patients with primary lung cancer who underwent lobectomy or segmentectomy with >ND1 lymph node dissection from 2008 to 2014 at Hachioji Medical Center of Tokyo Medical University. In total, 479 patients (294 males and 157 females) were examined. Cases of cerebral infarction occurring within 30 days of surgery were retrospectively studied. All surgeries were performed under mini-thoracotomy with thoracoscopy support. Vessels $>7 \mathrm{~mm}$ in diameter were amputated using a linear stapler. Results: Cerebral infarction occurred in four male patients, representing $0.8 \%$ (4/479) of all lung cancers and 1.4\% (4/294) of all male patients. Of these four patients, three were in their $60 \mathrm{~s}$ ( $1.4 \%$ of 157 patients in their $60 \mathrm{~s})$ and one was in his $70 \mathrm{~s}(0.5 \%$ of 215 patients in their $70 \mathrm{~s})$. Two cases involved adenocarcinomas, and two involved squamous cell carcinomas. One patient underwent right upper lobectomy, two underwent right lower lobectomy, and one underwent left upper lobectomy. The cerebral infarctions occurred in a branch of the vertebral artery. The pons was impaired in three cases, and the cerebellum was impaired in one. Three patients had pl2 disease, and one patient had pl3 disease. Operative time was $4-5 \mathrm{~h}$ in two cases, $5-6 \mathrm{~h}$ in one, and $>6 \mathrm{~h}$ in one. Only pl factor significantly differed between patients with and without postoperative cerebral infarction. Conclusions: To prevent cerebral infarction, the following factors should be considered: preoperative smoking cessation, operative positioning to protect the vertebral artery, shortening of operative time, and stronger anticoagulant therapy for high-risk patients, such as those with past history of transient ischemic attack.
\end{abstract}

Keywords: Cerebral Infarction, Lobectomy, Lung Cancer, Lymph Node Dissection, Pulmonary Vein Thrombosis

\section{Introduction}

Cerebral infarction occurs at a high frequency in patients undergoing left upper lobectomy (LUL) for lung cancer [16]. Thrombus caused by a long pulmonary vein (PV) stump is associated with an increased risk of cerebral infarction [1, 4-9]. In our clinical experience, cerebral infarction is not more frequent after LUL compared with other operative methods. We analyzed cases of surgery for lung cancer to identify factors that increase the risk of postoperative cerebral infarction.

\section{Methods}

Among patients with primary lung cancer who underwent lobectomy or segmentectomy with >ND1 lymph node dissection from 2008 to 2014, excluding preoperative chemotherapy cases, 479 patients were examined. There were 294 male and 157 female patients. Cerebral infarction cases occurring within 30 days after surgery were retrospectively studied. All surgeries were performed under minithoracotomy with thoracoscopy support. Vessels $>7 \mathrm{~mm}$ in diameter were amputated using a linear stapler.

Data were collected from medical records of patients at the 
Hachioji Medical Center of Tokyo Medical University with permission from the ethics committee (H-46). Written informed consent was waived because of the retrospective nature of this chart review.

Statistical analysis

Differences in clinical factors between patients with and without postoperative cerebral infarction were examined using analysis of variance. P values $<0.05$ were accepted as statistically significant.

\section{Results}

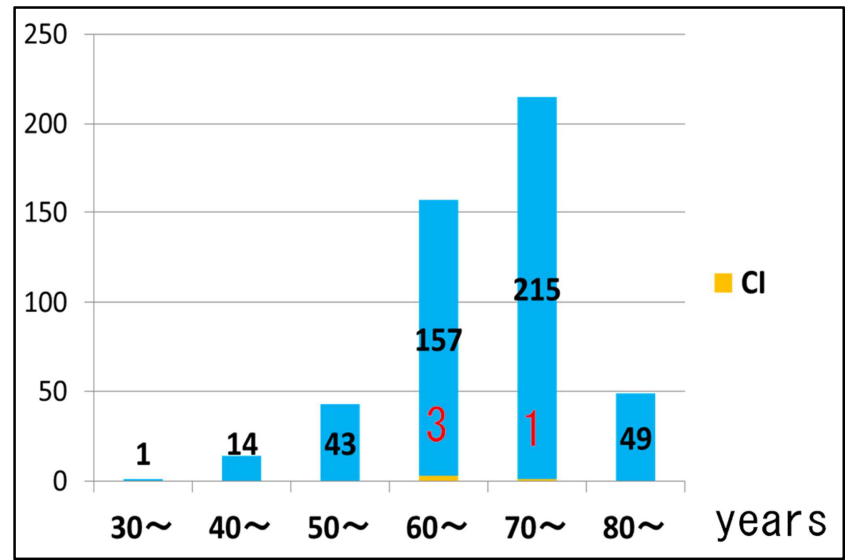

Figure 1. Relationship of age with postoperative cerebral infarction. CI: cerebral infarction.

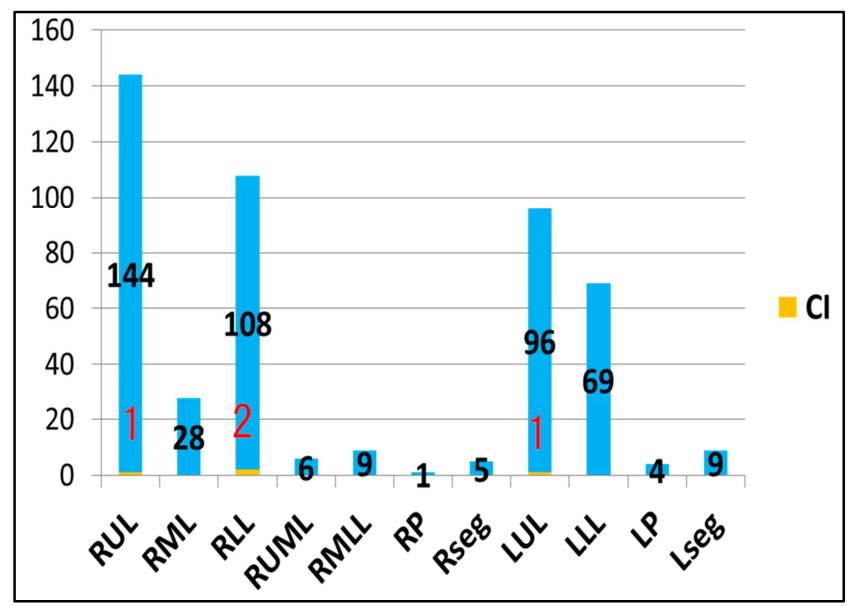

Figure 2. Postoperative cerebral infarction and operative methodology.

CI: cerebral infarction, RUL: right upper lobectomy, RML: right middle lobectomy, RLL: right lower lobectomy, RUML: right upper and middle lobectomy, RMLL: right middle and lower lobectomy, RP: right pneumonectomy, Rseg: right segmentectomy, LUL, left upper lobectomy, LLL, left lower lobectomy, LP: light pneumonectomy, Lseg: left segmentectomy.

Cerebral infarction occurred in four male patients, representing $0.8 \%$ (4/479) of all lung cancers and 1.4\% (4/294) of all male patients. Of these four patients, three were in their $60 \mathrm{~s}(1.4 \%$ of 157 patients in their $60 \mathrm{~s})$ and one was in his $70 \mathrm{~s}(0.5 \%$ of 215 patients in their 70s) (Figure 1). Two of these cases involved adenocarcinomas $(0.6 \%$ of 347 adenocarcinomas $)$, and the remaining two involved squamous cell carcinomas $(2.1 \%$ of 95 squamous cell carcinomas). One patient underwent right upper lobectomy (RUL, $0.7 \%$ of 144 RULs); two underwent right lower lobectomy (RLL, $1.9 \%$ of all RLLs), and one underwent left upper lobectomy (LUL, 1.0\% of 96 LULs) (Figure 2). Two patients were diagnosed with stage IB. disease $(1.3 \%$ of all 153 with stage IB), one with stage IIA disease $(2.0 \%$ of all 51 with stage IIA), and one with stage IIB disease (3.0\% of all 33 with stage IIB). In terms of tumor size, one was 10-20 $\mathrm{mm}$ in diameter $(0.8 \%)$, two were $20-30 \mathrm{~mm}$ in diameter $(1.5 \%)$, and one was $>70 \mathrm{~mm}$ in diameter $(7.1 \%)$. Three patients had pl2 disease $(2.5 \%)$, and one had pl3 disease (3.7\%) (Figure 3). Operative time was $4-5 \mathrm{~h}$ in two cases $(1.4 \%), 5-6 \mathrm{~h}$ in one $(1.4 \%)$, and $>6 \mathrm{~h}$ in one $(2.0 \%)$ (Figure 4). Only pl factor significantly differed between patients with and without postoperative cerebral infarction (Table 1).

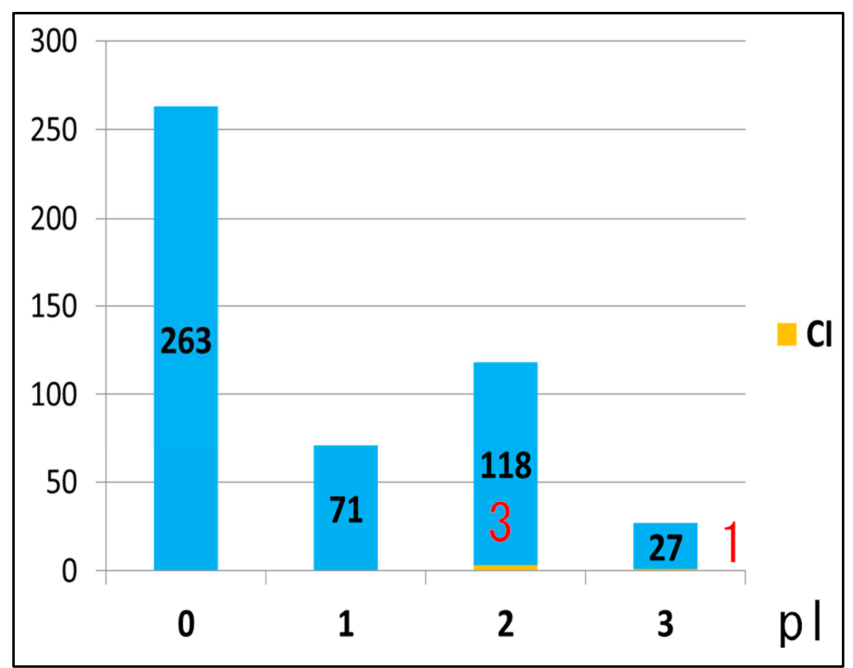

Figure 3. Postoperative cerebral infarction and degree of pleural invasion. CI: cerebral infarction.

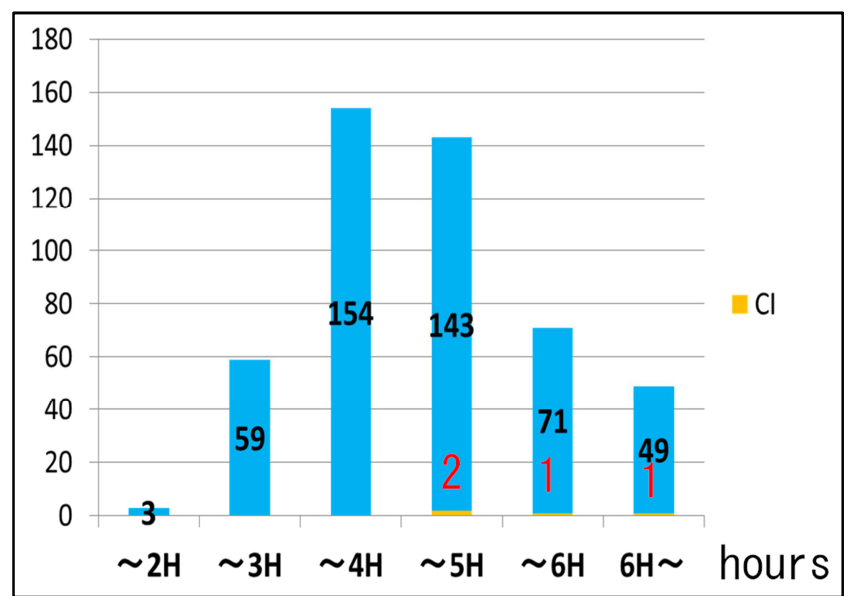

Figure 4. Postoperative cerebral infarction and operative time. CI: cerebral infarction. 
Table 1. Characteristics of patients with and without postoperative cerebral infarction.

\begin{tabular}{llll}
\hline Male/Female & $4 / 0$ & $290 / 185$ & 0.111 \\
$\leq 60$ ys/>60ys & $0 / 4$ & $58 / 417$ & 0.456 \\
ad/sq/others & $2 / 2 / 0$ & $345 / 93 / 37$ & 0.296 \\
OP methods & $1 / 0 / 2 / 0$ & $143 / 28 / 106 / 21$ & 0.867 \\
RU/RM/RL/other & $1 / 0 / 0$ & $95 / 68 / 13$ & \\
LU/LL/other & & & 0.569 \\
Size & $3 / 1$ & $290 / 185$ & \\
$<30 \mathrm{~mm} / \geq 30 \mathrm{~mm}$ & 0 & 26 & 0.645 \\
p-Stage & $0 / 2$ & $147 / 151$ & \\
0 & $1 / 1$ & $50 / 32$ & \\
IA/IB & $0 / 0$ & $62 / 3$ & 0.069 \\
IIA/IIB & 0 & 4 & 0.022 \\
IIIA/IIIB & $0 / 4$ & $216 / 259$ & \\
IV & & & \\
OP $\leq 4 \mathrm{H} />4 \mathrm{H}$ & $0 / 0 / 3 / 1$ & $263 / 71 / 115 / 26$ & \\
pl & & & \\
$0 / 1 / 2 / 3$ & & & \\
\hline
\end{tabular}

CI: cerebral infarction, ad: adenocarcinoma, sq: squamous cell carcinoma, OP: operation, RU: right upper lobectomy, RM: right middle lobectomy, RL: right lower lobectomy, LU: left upper lobectomy, LL: left lower lobectomy

Considering individual cases of postoperative cerebral infarction (Table 2), three were smokers and one was a current smoker. The pons was impaired in three cases, and the cerebellum was impaired in one.

Table 2. Clinical characteristics of patients with postoperative cerebral infarction.

\begin{tabular}{|c|c|c|c|c|c|c|c|c|c|c|c|}
\hline & Sex & Age & Op & Path & SI & Stage & POD & PH & Site & Note & outcome \\
\hline 1 & $\mathrm{M}$ & 69 & RLL & Ad & 1020 & 1B & 1 & App. & Pons & & 71.2m Alive PS0 \\
\hline 2 & M & 63 & RLL & $\mathrm{Sq}$ & 860 & $2 \mathrm{~A}$ & 4 & Colon ca. Pneumothorax & Cerebellum & & 9.1m Dead \\
\hline 3 & M & 76 & RUL & $\mathrm{Sq}$ & 800 & $2 \mathrm{~B}$ & 11 & Gastric ca. & Pons & Current smoker & $2.2 \mathrm{~m}$ Dead \\
\hline 4 & M & 68 & LUL & $\mathrm{Ad}$ & 0 & $1 \mathrm{~B}$ & 1 & AP TIA Subdural hematoma & Pons & Heparinization & $21.2 \mathrm{~m}$ Alive PS4 \\
\hline
\end{tabular}

M: male, OP: operative methods, RLL: right lower lobectomy, RUL: right upper lobectomy, LUL: left upper lobectomy, Path: pathology, ad: adenocarcinoma, sq: squamous cell carcinoma, SI: smoking index, POD: post-operative day, PH: past history, App: appendectomy, ca.: carcinoma, AP: angina pectris, TIA: transient ischemic attack, m: months

Two cases had a past history of malignant disease. Despite being a nonsmoker, one case had a past history of transient ischemic attack (TIA), angina pectoris, and subdural hematoma. Therefore, heparinization was perioperatively performed for this patient. One patient died 2.2 months after surgery, and one died 9.1 months after surgery. One patient completely recovered without complications, and the other was alive 21.2 months after surgery with loss of consciousness.

\section{Discussion}

Thrombosis in the PV stump can cause cerebral infarction and/or thrombosis in other vital organs [10-11]. Ohtaka et al. [7] reported that among 193 patients with primary lung cancer who underwent lobectomy, thrombus developed in the PV stump in seven (3.6\%) patients. Amazingly, all patients with thrombus had undergone LUL; this group of patients accounted for $13.5 \%$ of all LULs. These findings suggest that the length of the left superior PV stump is involved in thrombus formation. After lobectomy, the length of the left superior PV stump is longer than that of any other PV stump. Yamamoto et al. [1] reported six cases of cerebral infarction out of 562 lobectomies for lung cancer, including five patients who underwent LUL. Hattori et al [6] reported 10 patients with cerebral infarction out of 1670 patients who underwent lobectomy or more intervention. Among them, 5 patients underwent LUL. According to our results, postoperative cerebral infarction is not always frequent after LUL but was frequent after RUL. Because there were only a few cases, we could not sufficiently compare between patients with and without postoperative cerebral infarction. Therefore, only pleural invasion was significantly different between the groups. In each of these four cases, cerebral infarction occurred in a branch of the vertebral artery. One of three cases with basilar artery infarction died, and one had severe conscious disturbance. One patient with cerebellar infarction died within approximately 9 months postoperatively. Because the vertebral artery ascends in the intervertebral foramen, it narrows as it posteriorly bends, thereby facilitating thrombus formation. There may have been an issue with the intraoperative neck positioning in the cases included in the present study. These patients with cerebral infarction were elderly men; one was a current smoker, which may have increased the risk of cerebral infarction, and another patient had no history of smoking but had a history of TIA. Cerebral infarction occurred despite preventive therapy with heparin. Nakano et al. [12] also reported cases with postoperative cerebral infarction after lobectomy despite using anticoagulant therapy.

Yamamoto et al. [1] reported no relationship between operative time and the risk of cerebral infarction. However, 
increased operative time indicates increased time in the lateral position, thereby increasing the risk of cerebral infarction. Pleural invasion and adhesion to the chest wall also lead to excessive operative time. Cerebral infarction developed after 2013 in three of four cases. The surgical wound decreases in size every year. The angle at which the automatic stapler was used on the PV may also have increased the risk of cerebral infarction. The PV may be ligated at the bottom via traditional ligation methods. However, in the context of video-assisted thoracic surgery, the PV can be amputated only at a certain angle and position. Although ligation is performed in the same plane as the surface of the vascular intima, the stapler penetrates through the vascular intima. These factors may also contribute to thrombus formation.

We recommend that physicians seeking to prevent cerebral infarction should consider the following factors: preoperative smoking cessation, use of an operative position that protects the vertebral artery, shortening operative time, and use of stronger anticoagulant therapy for high-risk patients, such as those with a past history of TIA.

\section{Conclusions}

To prevent cerebral infarction, the following factors should be considered: preoperative smoking cessation, operative positioning to protect the vertebral artery, shortening of operative time, and stronger anticoagulant therapy for highrisk patients, such as those with past history of transient ischemic attack.

\section{Disclosure Statement}

All the authors do not have any possible conflicts of interest.

\section{References}

[1] Yamamoto T, Suzuki H, Nagato $K$, Nakajima T, Iwata $T$, Yoshida S, Yoshino I. Is left upper lobectomy for lung cancer a risk factor for cerebral infarction? Surg. Today. 2016; 46: $780-4$.

[2] Miyoshi R, Nishikawa S, Tamari S, Noguchi M, Hijiya K,
Chihara K. Pulmonary vin thrombosis after lobectomy with vein stump closure by ligation. Asian cardiovasc Thorac Ann. 2018; 26: 546-551.

[3] Kobayashi Y, Yashikozawa H, Takamatsu R, Watanabe R, Hoshi K, Ishii W, Sato S. Left upper lung lobectomy is an embolic risk factor for cerebral infarction. J Storoke Cerebrovasc Dis. 2017; 26: e177-e179.

[4] Ohtaka K, Hida H, Kaga K, Takahashi Y, Kawase H, Hayama S, Ichimura T, Senmaru N, Honma N, Matsui Y. Left upper lobectomy can be a risk factor for thrombus in the pulmonary vein stump. J Cardiovasc Surg. 2014; 6: 9: 5.

[5] Usui G, Matsumoto J, Hashimoto H, Katano T, Kusakabe M, Horiuchi H, Okubo S. Thrombus reformation in the pulmonary vein stump confirmed 16 months after cerebral embolism on the day after left upper lobectomy for lung cancer. J Storoke Cerebrovasc Dis. 2018; 27: e225-e227.

[6] Hattori A, Takamochi K, Kitamura Y, Matsunaga T, Suzuki K, Shiaki Oh, Suzuki K. Risk factor analysis of cerebral infarction and clinicopathological characteristics of left upper pulmonary vein stump thrombus after lobectomy. Gen. Thorac. Cardiovasc. Surg. 2019; 67 (2); 247-253.

[7] Ohtaka K, Hida Y, Kaga K, Kato T, Muto J, Nakada-Kubota $\mathrm{R}$, Sasaki T, Matsui Y. Thrombosis in the pulmonary vein stump after left upper lobectomy as a possible cause of cerebral infarction. Ann. Thorac. Surg. 2013; 95: 1924-9.

[8] Ichimura H, Ozawa Y, Nishina H, Shiotani S. Thrombus formation in the pulmonary vein stump after left upper lobectomy: a report of four cases. Ann. Thorac. Cardiovasc. Surg. 2014; 20 Suppl: 613-6.

[9] Kobayashi Y, Yahikozawa H, Takamatsu R, Watanabe R, Hoshi K, Ishii W, Sato S. Left Upper Lung Lobectomy Is an Embolic Risk Factor for Cerebral Infarction. J. Stroke Cerebrovasc. Dis. 2017; 26 (9); e177-e179.

[10] Asteriou C, Barbetakis N, Efstathiou A, Kleontas A, Tsilikas C. Renal artery thrombosis following lobectomy for lung cancer. Case Rep Oncol. 2010; 3 : 08-211.

[11] Umeda Y, Matsumoto S, Mori Y, Takiya H. Postoperative superior mesenteric artery and cerebral infarction possibly due to the thrombus at the left superior pulmonary vein stump. Kyobu Geka. 2015; 68: 967-969. In Japanese.

[12] Nakano T, Inaba $M$, Kaneda $H$. Recurrent cerebral attack caused by thrombosis in the pulmonary vein stump in a patient with left upper lobectomy on anticoagulant therapy: case report and literature review. Surg. Case Rep. 2017; 3: 101. 